\title{
Preventing and controlling tuberculosis among refugees in Europe: more is needed
}

\author{
To the Editor:
}

We read with interest the study by DE VRIES et al. [1] reporting on the results of tuberculosis screening among recently arrived migrants and refugees in the Netherlands.

This study brings several issues for debate in a moment where migration to Europe generates concerns and potential risks for stigmatisation and discrimination. In 2015, >1 million migrants and refugees reached Europe by different routes, more than four times more people than in 2014 (when there were only 219000) $[2,3]$.

Migrants and refugees are among the most vulnerable groups for tuberculosis infection and disease, due to multiple reasons including their precarious living and travelling conditions. In addition, they are often coming from countries with higher tuberculosis incidence than the host countries [4-6]. In addition to being a human right, adequate management of tuberculosis and latent tuberculosis infection (LTBI) in migrants and refugees is important to optimise tuberculosis control and elimination strategies in Europe, as tuberculosis does not respect borders [4-6]. It is important to underline that the increasing number of arrivals makes it more and more difficult to implement adequate tuberculosis screening and treatment strategies in centres hosting migrants/refugees or countries through which they are transiting.

A challenge in developing and adapting evidence-based screening policies is a lack of information on the exact number of migrants/refugees to be screened. Health authorities in the Netherlands decided, rationally, to evaluate the yield of screening for refugees coming from countries with an incidence $<50$ per 100000 inhabitants to evaluate whether previous decisions had to be confirmed or modified. In the Dutch setting, the yield of screening proved to be rather low among Syrian refugees and other asylum seekers: the tuberculosis prevalence of screening in this population was 26 per 100000 population, meaning that 3787 individuals need to be screened to identify one tuberculosis case. 12 cases of pulmonary tuberculosis were diagnosed (seven from Syria), four being sputum smear positive and the others culture positive.

In our opinion, two interesting pieces of information need to be discussed. 1) Four individuals with an originally negative chest radiography developed tuberculosis in the following 6 months. The information on their LTBI status was not reported [1]. If tuberculosis elimination has to be reached, we probably need to become more "aggressive" in our prevention approach, focusing on LTBI diagnosis and treatment [5-7]. Although some refugees originate from relatively low tuberculosis incidence countries, they may have been infected during their joint travel and accommodation with refugees coming from high tuberculosis incidence countries. LTBI may turn into disease after arriving in the host countries. 2) The importance of ensuring universal access to tuberculosis services is confirmed by the self-reporting of a tuberculosis case after screening discontinuation [1].

The findings of this study show how important is, at the national level, to base decisions on evidence, to ensure proper surveillance, and to monitor/evaluate quality data on migrants and refugees. There is a need for improved surveillance and cross-border data exchange for those refugees moving from one country to another.

However, the findings of this study cannot be automatically extended to other settings, as the profile of refugees reaching northern Europe may differ from that observed in other European countries.

The recently approved tuberculosis elimination framework [5], which includes eight areas for action, calls for more efforts by national programmes to target LTBI as well as tuberculosis disease, within the framework of an integrated European effort to coordinate policies and share best practices.

More evidence is needed on the screening policies in Europe, as little is known and from only a few countries [8-10].

Most of the evidence (table 1) results from a systematic review of the screening policies performed in the European Union [9]. Overall, the coverage of screening practices was higher in asylum seekers (94\%) than in other migrants $(47.8 \%)$, with a median yield of $0.30 \%$ and $0.176 \%$, respectively. In Switzerland [10], the yield of screening based on routine chest radiography (14.3 per 10000) was only slightly higher than that achieved with individual assessment (12.4 per 10000), based on an integrated scoring system capturing different criteria including the tuberculosis prevalence in the country of origin, personal and family tuberculosis history, and symptoms/general conditions as assessed by the interviewing nurse. 
TABLE 1 Screening practices and yield of screening in selected European countries

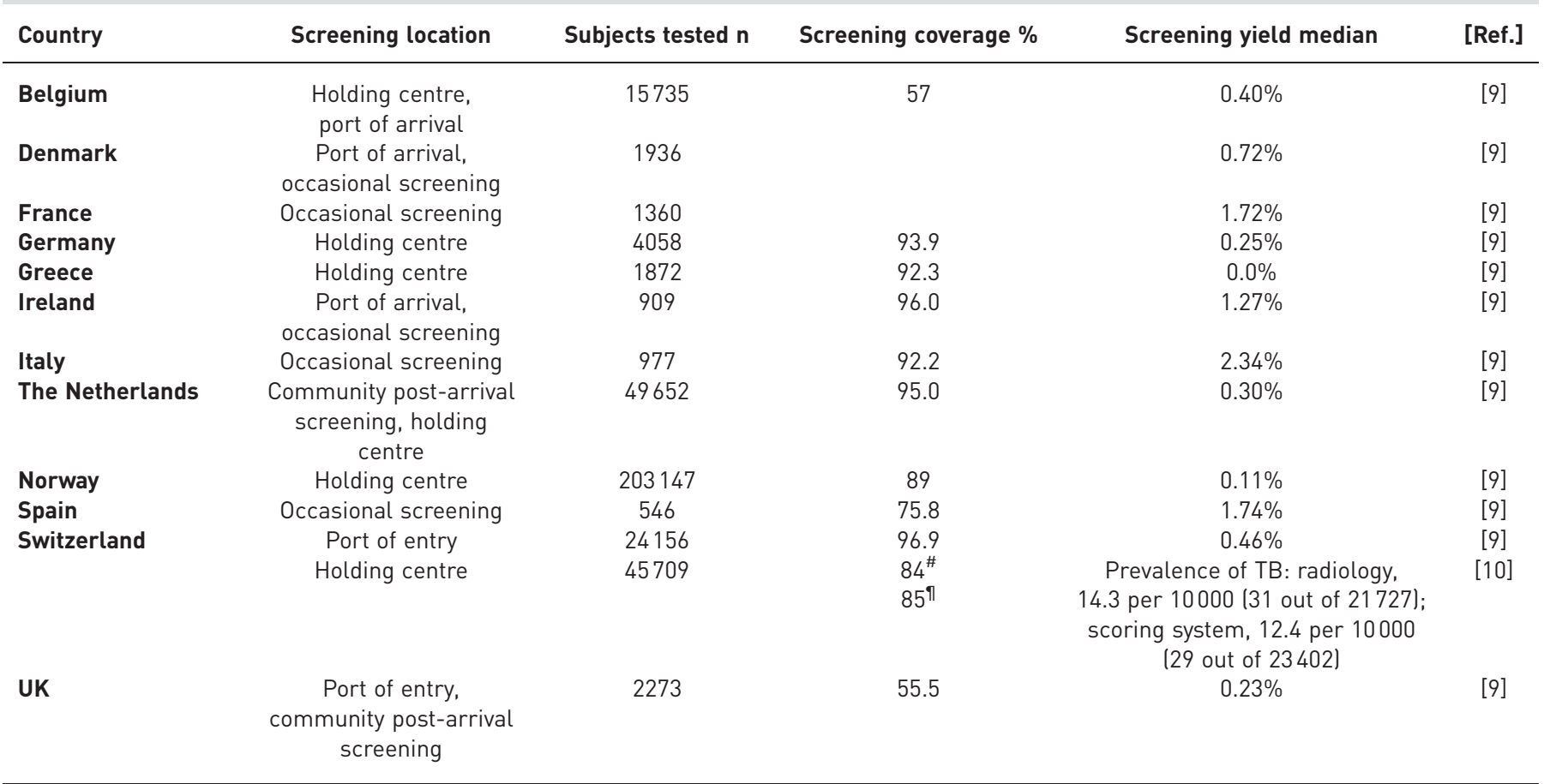

TB: tuberculosis. " : 2004-2005; ๆः: 2007-2008.

The few studies available [8-10] show that different screening policies and practices are implemented in Europe, chest radiography being common in all countries within differently organised algorithms that include symptom evaluation, bacteriology and, in some countries, LTBI diagnosis via the tuberculin skin test and/or interferon- $\gamma$ release assays.

While $22(71 \%)$ countries recently reported screening for LTBI in high-risk groups (which include asylum seekers and migrants), only six countries (Greece, the Netherlands, Portugal, the Former Yugoslav Republic of Macedonia, Slovakia and Switzerland) were able to report LTBI treatment completion rates, which ranged between $40 \%$ and $88 \%$ [9].

Improved surveillance and further studies are needed to ensure that quality diagnosis and treatment for tuberculosis and LTBI (when feasible) are provided to all migrants and refugees in Europe.

0 @ERSpublications

A coordinated effort is needed in Europe to ensure adequate diagnosis and treatment of tuberculosis among refugees http://ow.ly/YEfXp

Masoud Dara ${ }^{1}$, Ivan Solovic ${ }^{2}$, Delia Goletti ${ }^{3}$, Giovanni Sotgiu ${ }^{4}$, Rosella Centis ${ }^{5}$, Lia D’Ambrosio ${ }^{5,6}$, Brian Ward ${ }^{7}$, Vitor Teixeira $^{7}$, Christina Gratziou ${ }^{8}$ and Giovanni Battista Migliori ${ }^{5}$

${ }^{1}$ World Health Organization Office at the European Union, Brussels, Belgium. ${ }^{2}$ National Institute for TB, Lung Diseases and Thoracic Surgery, Catholic University Ruzomberok, Vysne Hagy, Slovakia. ${ }^{3}$ Translational Research Unit, National Institute for Infectious Diseases, L. Spallanzani, Rome, Italy. ${ }^{4}$ Clinical Epidemiology and Medical Statistics Unit, Dept of Biomedical Sciences, University of Sassari - Research, Medical Education and Professional Development Unit, AOU Sassari, Sassari, Italy. ${ }^{5}$ WHO Collaborating Centre for TB and Lung Diseases, Fondazione S. Maugeri, Tradate, Italy. ${ }^{6}$ Public Health Consulting Group, Lugano, Switzerland. ${ }^{7}$ EU Affairs Dept, European Respiratory Society, Brussels, Belgium. ${ }^{8}$ University Respiratory Medicine Unit, Evgenidio Hospital, Athens, Greece.

Correspondence: Giovanni Battista Migliori, World Health Organization Collaborating Centre for Tuberculosis and Lung Diseases, Fondazione S. Maugeri, Care and Research Institute, Via Roncaccio 16, 21049, Tradate, Italy.

E-mail: giovannibattista.migliori@fsm.it

Received: Feb 132016 | Accepted: Feb 152016

C. Gratziou is the ERS Advocacy Council Chair and Secretary for EU Affairs 2015-2018.

Conflict of interest: B. Ward and V. Teixeira are employees of the European Respiratory Society. 


\section{References}

1 de Vries G, van Rest J, Meijer W, et al. Low yield of screening asylum seekers from countries with a tuberculosis incidence of <50 per 100000 population. Eur Respir J 2016; 47: 1870-1872.

2 Matteelli A, Lönnroth K, Mosca D, et al. Cameroon's multidrug-resistant tuberculosis treatment programme jeopardised by cross-border migration. Eur Respir J 2016; 47: 686-688.

3 United Nations High Commission for Refugees. Refugees and migrants crossing the Mediterranean to Europe. Overview of arrival trends as of 31 August 2015. http://data.unhcr.org/mediterranean/regional.php Date last accessed: February 4, 2016.

4 Dara M, de Colombani P, Petrova-Benedict R, et al. The minimum package for cross-border TB control and care in the WHO European Region: a Wolfheze consensus statement. Eur Respir J 2012; 40: 1081-1090.

5 Lönnroth K, Migliori GB, Abubakar I, et al. Towards tuberculosis elimination: an action framework for low-incidence countries. Eur Respir J 2015; 45: 928-952.

6 Getahun H, Matteelli A, Abubakar I, et al. Management of latent Mycobacterium tuberculosis infection: WHO guidelines for low tuberculosis burden countries. Eur Respir J 2015; 46: 1563-1576.

7 D'Ambrosio L, Dara M, Tadolini M, et al. Tuberculosis elimination: theory and practice in Europe. Eur Respir J 2014; 43: 1410-1420.

8 Coker R, Bell A, Pitman R, et al. Tuberculosis screening in migrants in selected European countries shows wide disparities. Eur Respir J 2006; 27: 801-807.

9 Klinkenberg E, Manissero D, Semenza JC, et al. Migrant tuberculosis screening in the EU/EEA: yield, coverage and limitations. Eur Respir J 2009; 34: 1180-1189.

10 Schneeberger Geisler S, Helbling P, Zellweger JP, et al. Screening for tuberculosis in asylum seekers: comparison of chest radiography with an interview-based system. Int J Tuberc Lung Dis 2010; 14: 1388-1394.

\section{Preventing and controlling tuberculosis among refugees in Europe: more needed for high-risk populations}

\section{From the authors:}

We thank M. Dara and colleagues for their commentary on our article "Low yield of screening asylum seekers from countries with a tuberculosis (TB) incidence of $<50$ per 100000 population" [1]. We agree that the current high influx of refugees from both low- and high-incidence countries into countries that already have a TB incidence $<10$ per 100000 population, e.g. many European Union member states, brings up a number of new challenges.

We agree with M. Dara and colleagues that profiles of migrating populations differ, as determined for example by country of origin or reasons for migration, such as study, employment, family reunification or war. In the Netherlands, screening processes differ for recently arrived migrants and refugees and the effectiveness of these interventions is evaluated separately. Results of chest radiography screening of (regular) migrants were previously analysed for the period 2005-2010 [2]. Overall, 108 TB cases were diagnosed among 117389 screened migrants, resulting in a TB prevalence of 92 per 100000 screened persons (95\% CI 75-109). Only seven cases were identified by screening of 31218 migrants from countries with a TB incidence of $<50$ per 100000 , resulting in a TB prevalence of 22 per 100000 screened persons (95\% CI 9-46) and a number needed to screen (NNS) of 4460 . We would like to reiterate that the Netherlands uses a threshold of 2000 for NNS, in order to limit exposure of healthy individuals to radiation. The low yield among (regular) migrants from countries with a TB incidence of $<50$ per 100000 led to the advice to the Ministry of Health to limit screening to migrants from countries with an estimated TB incidence of $>50$ per 100000 population. This has been in effect since January 1, 2015. Our current evaluation of screening asylum seekers ( January 2011-September 2015) from countries with a TB incidence of $<50$ per 100000 had similar results, i.e. a TB prevalence of 26 per 100000 screened persons (95\% CI 14-45) and a NNS of 3787, and the same consideration led to the advice to the Ministry of Health to stop screening. We agree that our results cannot be automatically extrapolated to other countries and would be interested to read other countries' experiences. However, our results are not unexpected in asylum seekers from countries with traditionally a low TB incidence. Even if their circumstances dramatically change and they are staying in densely populated camps, there will initially still be few cases that transmit the disease. 